\title{
Composition en acides gras, fermeté et coloration du tissu gras sous-cutané des carcasses d'agneaux
}

\author{
Gérard Bozzolo*, Michel Bouillier-Oudot
}

École nationale supérieure agronomique (Ensat), avenue de l'agrobiopole, BP 107, Auzeville-Tolosane, 31326 Castanet-Tolosan cedex, France

(Reçu le 12 mars 1998 ; accepté le 6 avril 1998)

\begin{abstract}
Fatty acids composition of layer fat from lamb's carcasses and its firmness and colour characteristics. Fatty acid (F.A.) composition of subcutaneous fat sampled on Lacaune lamb's carcasses was determined by gas chromatography to evaluate the relationship with firmness and colour. Four classes of firmness from very hard layer fat up to very soft and oily fat $(n=4 \times 20)$ and seven classes of colour adjusted with Munsell's toner $(n=7 \times 16)$ were investigated. Very hard layer fat contains more even saturated chain F.A. (35.6\% versus $27.5 \%, P<0.05 ; \eta=0.78$ ) than soft and oily fat. These are mostly long chain fats: C16 and C18. In the opposite the concentration of odd and/or branched chain F.A. is higher in soft fatty tissues $(10.2 \%$ versus $5.6 \%, P<0.05$; $\eta=0.72$ ). In this category, IC13 and $\mathrm{C} 15$ and AIC17 are the most linked $\eta=0.75, \eta=0.74, \eta$ $=0.72$ respectively). The group of monoenoic F.A. is less discriminating than former ones $(\eta=0.53)$ except for one difference between very hard fat and others $(53.5 \%$ versus $55.7 \%, P<0.05)$. Therefore, oleic acid is like a long chain acid with its intermediate melting point (cis-and trans-forms) and it is more present in firmest fats ( $46 \%$ versus $42 \%, P<0.05$ ). In contrast $C 11=1$ and $C 12=1$ and $\mathrm{C} 16=1$ are better connected with soft oily tissues $(\eta=0.75, \eta=0.80, \eta=0.72$ respectively) but they are present in small amounts. In this way average concentrations are well balanced between the different classes of firmness $(14.6 \%, 12.2 \%, 10.4 \%, 7.6 \%$ from very soft and oily up to very firm fat, $P<0.001)$. Colour is less dependant on fatty acid composition. The strongest difference for long chain F.A. is found between clear colours (white and cream white) and brown red colour (C18 + C18 $=$ $1: 55.1 \%$ versus $48.5 \%, P<0.01 ; \eta=0.53$ ). With odd number and/or branched chain F.A. the tendency is inverted $(6.8 \%$ versus $11.8 \% ; \eta=0.61)$. The higher moisture of light swarthy carcasses $(24.5 \%$ versus $20.4 \%, P<0.05)$ and their lower fattening grade will be a consequence of post mortem autoxidation during carcass shrinkage. But first of all, fatty acid composition is poorly determined and this supposes that other sources of explanation will need to be investigated. The observations realised on 570 carcasses show that white fats are very often associated with firm layer fat and that other colours are randomly distributed over the firmness classes. This strengthens this point of view. (c) Elsevier / Inra
\end{abstract}

lamb / carcass / firmness / colour / fatty acids

\footnotetext{
* Correspondance et tirés à part
} 
Résumé - La fermeté (quatre classes, $n=4 \times 20$ ) et la couleur (sept classes appréciées à l'aide du nuancier Munsell, $n=7 \times 16$ ) du gras externe de carcasses d'agneaux de boucherie Lacaune sont mises en relation avec la composition en acides gras (A.G.) du tissu gras sous-cutané. Les gras fermes sont plus riches en A.G. saturés pairs que les gras mous et huileux $(35,6 \%$ vs $27,5 \%, p<0,05$; $\eta=0,78$ ), notamment en acides gras longs C16 et C18. C'est l'inverse pour l'ensemble des A.G. impairs et/ou ramifiés $(5,6 \%$ vs $10,2 \%, p<0,05 ; \eta=0,72)$ en particulier IC13 $(0,1 \%$ vs $0,3 \%$, $\eta=0,75), \mathrm{C} 15(0,9 \%$ vs $2 \%, \eta=0,74)$ et $\mathrm{AIC} 17(0,8 \%$ vs $1,4 \%, \eta=0,72)$. En revanche, le regroupement des A.G. mono-insaturés est peu discriminant $(\eta=0,53)$ si ce n'est pour les gras très fermes qui se distinguent des autres $(53,5 \%$ vs $55,7 \%, p<0,05)$. En effet, parmi ceux-ci, C18 $=1$, présent en forte quantité ( $46 \%$ vs $42 \%$ ) intervient avec un point de fusion permettant le durcissement des gras après ressuage, alors que les A.G. très discriminants : $\mathrm{Cl} 1=1, \mathrm{C} 12=1, \mathrm{Cl} 16=1(\eta=0,75$, $\eta=0,8, \eta=0,72$ respectivement) sont représentés en faible proportion. En excluant $\mathrm{C} 18=1$ cette somme caractérise bien, alors, les différences de classes de fermeté $(14,6 \%, 12,2 \%, 10,4 \%, 7,6 \%$ respectivement de très mou et huileux à très ferme, $p<0,001$ ). La couleur est peu influencée par la composition en A.G. Les différences les plus marquées entre teneurs en A.G. concernent l'opposition entre nuances claires (blanc et blanc crème) et la nuance brun-rouge vis-à-vis des A.G. longs (C18 + $\mathrm{C} 18=1: 55,1 \%$ vs $48,1 \%, p<0,001 ; \eta=0,53)$ et de la somme des A.G. impairs ev/ou ramifiés $(6,8 \%$ vs $11,8 \%, \eta=0,61$ ). Cependant, la composition en A.G. reste peu discriminante et suggère que d'autres facteurs que leur genèse sont à rechercher. La plus forte teneur en eau pour la coloration bistre clair $(24,5 \%$ vs $20,4 \%, p<0,05)$ ainsi que le moindre état d'engraissement externe des carcasses suggère l'implication de réactions d'auto-oxydation pendant la phase de ressuage pour cette teinte. Les observations réalisées sur 570 carcasses montrent une tendance des gras blancs à être, dans la majorité des cas, fermes à très fermes. Cependant, la dispersion selon les classes de fermeté reste imprécise pour les autres colorations, ce qui confirme la faible liaison entre couleur et composition en A.G. de la graisse de couverture des carcasses. (C) Elsevier /Inra

agneau / carcasse / gras / fermeté / couleur / acides gras

\section{INTRODUCTION}

Le commerce des carcasses d'agneaux fait de plus en plus référence à des critères de qualité sous la pression des différents intervenants de la filière. À côté du classement officiel (classement EUROP pour la conformation, degré d'engraissement, poids de la carcasse), la fermeté et la couleur du gras de couverture des carcasses constituent des qualités officieuses retenues dans la démarche commerciale, notamment pour les agneaux labellisés. Le lien entre la fermeté et la composition des triglycérides en acides gras (A.G.) du tissu gras sous-cutané est assez bien connu $[8,14,19,20]$ Cependant, les résultats exprimés portent, pour la majorité des comparaisons, sur un nombre réduit d'échantillons ou sur un nombre de classes de fermeté restreint.
Pour la coloration, les origines et causes sont moins bien appréhendées et présentent un caractère multifactoriel $[4,22,24]$. Les résultats de Zhou et al. [28], obtenus chez les bovins, semblent indiquer une liaison étroite entre le rapport A.G. mono-insaturés/ A.G. saturés et la couleur jaune du gras externe des carcasses. Chez les ovins, l'ampleur du problème des défauts de coloration observés sur carcasse $[12,16,22]$ méritait de préciser ce support éventuel que peut constituer la composition en A.G. du tissu gras sous-cutané.

Nous rapportons les résultats de composition en A.G. des échantillons de tissu gras prélevés lors d'essais alimentaires réalisés avec des agneaux de race Lacaune. Les résultats zootechniques et les caractéristiques de carcasse ont été antérieurement publiés $[5,6]$. 


\section{MATÉRIEL ET MÉTHODES}

Les agneaux, de type roquefort, étaient sevrés précocement (4 à 5 semaines) et engraissés intensivement en bergerie (centre d'engraissement du Gebro). Ils ont été nourris avec un aliment de finition granulé (UFV $\cdot \mathrm{kg}^{-1}$ de M.S. : 0,97 ; M.A.T. : $165 \mathrm{~g} \cdot \mathrm{kg}^{-1}$ de M.S., Cellulose brute : $79 \mathrm{~g} \cdot \mathrm{kg}^{-1}$ de M.S.) distribué à volonté et complété par de la paille en libre accès. Un total de 570 carcasses ont été examinées et annotées. Les échantillons de tissu gras sous-cutané ont été prélevés latéralement à la base de la queue après $12 \mathrm{~h}$ de ressuage des carcasses.

Les notations de fermeté et de couleur du tissu gras de couverture ont été effectuées dans le même abattoir par un opérateur unique expérimenté, selon des barèmes déjà définis $[3,4]$ faisant référence à quatre classes de fermeté (Ten1 : gras mou et huileux, Ten 4 : gras ferme et caillé) et à sept classes de coloration (Coull : blanc, Coul2 : blanc crème, Coul3 : rosée, Coul 4 : bistre clair, Coul 5 : brun-rouge, Coul6 : jaune clair, Coul7 : bistre clair avec reflets verdâtres) correspondant aux teintes du nuancier Munsell (respectivement 5YR9/4, 7.5YR9/4, 5YR8/6, 7.5YR8/6, 5YR8/8, 10YR9/4, 10YR8/6).

Pour permettre d'intégrer à ces observations le degré d'engraissement des carcasses, la note de gras de couverture (NGext), appréciée selon les critères du classement Europa, et l'épaisseur du gras dorsal (EGdo), mesurée en mm au niveau des $4^{c}$ et $5^{\mathrm{e}}$ vertèbres lombaires, sont également introduits.

L'extraction des lipides a été réalisée selon la procédure de Folch et al. [13] et la composition en A.G. déterminée par chromatographie en phase gazeuse à partir des esters méthyliques selon un protocole décrit précédemment [4].

\subsection{Traitement des résultats}

Nous avons constitué les échantillons de façon à établir des comparaisons sur la base d'effectifs intra-classe équilibrés. Ceux-ci ont été ajustés en retenant l'effectif de la classe la plus faiblement représentée et en procédant par tirage aléatoire pour constituer les éléments des autres classes. Dans le cas de l'étude de la relation entre la fermeté du gras sous-cutané et sa composition en A.G., nous avons pu retenir 20 prélèvements par classe de notation, soit un total de 80 analyses. En ce qui concerne l'étude de la relation entre la coloration et la composition en A.G.,
16 prélèvements par note de coloration ont été rassemblés, soit 112 analyses.

La liaison entre composition en A.G. et fermeté ou couleur est abordée par la comparaison des moyennes (test de Newman-Keuls) après analyse de la variance. Le rapport de corrélation $\eta$ [11] est utilisé pour préciser l'intensité de la liaison entre notation et composition en A.G. Une analyse discriminante (AD) permet de préciser l'efficacité de la séparation entre notes sur ces critères et appuie l'observation synthétique des données apportée par l'analyse factorielle des correspondances (AFC) établies à partir des profils d'acides gras.

L'étude des relations entre notes de fermeté et types de coloration fait appel à l'ensemble des 570 jugements effectués sur carcasses. La liaison entre les deux caractères est observée en $\mathrm{AFC}$ pour mettre en évidence les proximités entre ces deux critères d'appréciation de la qualité des carcasses.

\section{RÉSULTATS ET DISCUSSION}

\subsection{Fermeté du tissu gras externe}

À l'exception des A.G. : C10, IC17, $\mathrm{C} 18=2, \mathrm{C} 18=3, \mathrm{C} 18=4, \mathrm{C} 20=1$, les A.G. détectés présentent des teneurs significativement différentes selon les classes de fermeté ( $p<0,05 ;$ tableau $I)$. Si l'on se réfère aux acides gras quantitativement les plus représentés (C14, C16, C16 $=1, \mathrm{C} 17$, $\mathrm{C} 17=1, \mathrm{C} 18, \mathrm{C} 18=1, \mathrm{C} 18=2$, et dont la proportion dans le tissu gras est au moins supérieure à $3 \%$ dans l'une des classes de fermeté), il apparaît que les graisses les plus molles sont significativement associées aux teneurs en A.G. : C14, C16, C18, C18 = 1 les plus faibles et, à l'inverse, aux teneurs les plus élevées en acides gras : C16 $=1$, $\mathrm{C} 17=1, \mathrm{C} 17$.

Les A.G. les plus liés à la notation $(\eta>0,71)$ sont : $\mathrm{C} 11=1, \mathrm{C} 12=1, \mathrm{IC} 13$, $\mathrm{C} 15, \mathrm{C} 16=1, \mathrm{AlC} 17, \mathrm{C} 17=1, \mathrm{IC} 17=1$, IC18, C18. À l'exception de C18, tous ces acides gras ont pour caractéristique de présenter des teneurs significativement plus élevées pour les gras très mous (Ten 1 ) et plus faibles pour les gras les plus fermes (Ten4). 
Tableau I. Composition en acides gras selon la fermeté du tissu gras sous-cutané.

\begin{tabular}{|c|c|c|c|c|c|c|}
\hline Fermeté & Ten 1 & Ten 2 & Ten 3 & Ten4 & $\eta$ & c.v. \\
\hline Effectif & 20 & 20 & 20 & 20 & & \\
\hline \multicolumn{7}{|l|}{ Acides gras } \\
\hline $\mathrm{C} 10$ & 0,22 & 0,23 & 0,21 & 0,22 & 0,12 & 0,29 \\
\hline $\mathrm{C} 10=1$ & $0,33^{a}$ & $0,29^{a b}$ & $0,24^{b}$ & $0,13^{c}$ & 0,71 & 0,34 \\
\hline $\mathrm{C} 11=1$ & $0,34^{a}$ & $0,22^{\mathrm{b}}$ & $0,12^{\mathrm{c}}$ & $0,03^{\mathrm{d}}$ & 0,75 & 0,63 \\
\hline $\mathrm{C} 12$ & $0,15^{\mathrm{b}}$ & $0,21^{\mathrm{a}}$ & $0,15^{\mathrm{b}}$ & $0,2^{\mathrm{ab}}$ & 0,35 & 0,43 \\
\hline $\mathrm{C} 12=1$ & $0,19^{\mathrm{a}}$ & $0,15^{\mathrm{b}}$ & $0,11^{\mathrm{c}}$ & $0,07^{\mathrm{d}}$ & 0,80 & 0,35 \\
\hline IC 13 & $0,31^{\mathrm{a}}$ & $0,25^{\mathrm{a}}$ & $0,17^{\mathrm{b}}$ & $0,06^{\mathrm{c}}$ & 0,75 & 0,48 \\
\hline $\mathrm{C} 13$ & $0,17^{a}$ & $0,12^{\mathrm{b}}$ & $0,08^{c}$ & $0,05^{\mathrm{d}}$ & 0,68 & 0,44 \\
\hline IC14 & $0,66^{a}$ & $0,42^{\mathrm{b}}$ & $0,25^{\mathrm{bc}}$ & $0,08^{c}$ & 0,58 & 0,87 \\
\hline C14 & $2,65^{\mathrm{b}}$ & $3,33^{\mathrm{a}}$ & $2,90^{\mathrm{b}}$ & $3,37^{\mathrm{a}}$ & 0,51 & 0,17 \\
\hline $\mathrm{C} 14=1$ & $2,37^{a}$ & $2,09^{a}$ & $1,76^{\mathrm{b}}$ & $1,18^{c}$ & 0,68 & 0,27 \\
\hline IC 15 & $0,51^{\text {i }}$ & $0,41^{\mathrm{b}}$ & $0,30^{\mathrm{c}}$ & $0,16^{\mathrm{d}}$ & 0,67 & 0,44 \\
\hline $\mathrm{AIC} 15$ & $0,46^{\mathrm{a}}$ & $0,37^{\mathrm{b}}$ & $0,28^{c}$ & $0,21^{\mathrm{d}}$ & 0,71 & 0,29 \\
\hline $\mathrm{C} 15$ & $1,98^{\mathrm{a}}$ & $1,59^{\mathrm{b}}$ & $1,23^{\mathrm{c}}$ & $0,93^{\mathrm{d}}$ & 0,74 & 0,26 \\
\hline $\mathrm{C} 15=1$ & $1,34^{a}$ & $0,79^{\mathrm{b}}$ & $0,50^{c}$ & $0,22^{\mathrm{d}}$ & 0,71 & 0,59 \\
\hline IC 16 & $0,75^{a}$ & $0,52^{\mathrm{b}}$ & $0,34^{\mathrm{c}}$ & $0,23^{c}$ & 0,70 & 0,45 \\
\hline $\mathrm{C} 16$ & $18,29^{c}$ & $20,02^{b}$ & $20,48^{\mathrm{b}}$ & $21,99^{a}$ & 0,69 & 0,07 \\
\hline $\mathrm{C} 16=1$ & $5,78^{a}$ & $5,48^{a}$ & $4,92^{\mathrm{b}}$ & $4,10^{c}$ & 0,72 & 0,13 \\
\hline IC 17 & 0,32 & 0,30 & 0,26 & 0,29 & 0,30 & 0,24 \\
\hline $\mathrm{AIC} 17$ & $1,37^{\mathrm{a}}$ & $1,23^{\mathrm{b}}$ & $1,04^{\mathrm{c}}$ & $0,82^{d}$ & 0,73 & 0,17 \\
\hline $\mathrm{C} 17$ & $3,46^{4}$ & $2,93^{\mathrm{b}}$ & $2,87^{\mathrm{b}}$ & $2,41^{\mathrm{c}}$ & 0,58 & 0,19 \\
\hline $\mathrm{C} 17=1$ & $3,86^{a}$ & $2,97^{\mathrm{b}}$ & $2,49^{c}$ & $1,67^{d}$ & 0,74 & 0,27 \\
\hline IC 18 & $0,66^{\mathrm{a}}$ & $0,47^{\mathrm{b}}$ & $0,37^{\mathrm{c}}$ & $0,24^{d}$ & 0,72 & 0,36 \\
\hline $\mathrm{C} 18$ & $6,15^{\mathrm{c}}$ & $6,68^{c}$ & $8,39^{\mathrm{b}}$ & $9,80^{\mathrm{a}}$ & 0,74 & 0,17 \\
\hline $\mathrm{C} 18=1$ & $41,77^{c}$ & $43,03^{b}$ & $45,13^{\mathrm{a}}$ & $45,88^{\mathrm{a}}$ & 0,58 & 0,05 \\
\hline $\mathrm{C} 18=2$ & 4,44 & 4,49 & 4,10 & 4,28 & 0,22 & 0,16 \\
\hline C19 & $0,21^{\mathrm{a}}$ & $0,18^{\mathrm{b}}$ & $0,18^{b}$ & $0,16^{\mathrm{h}}$ & 0,45 & 0,17 \\
\hline $\mathrm{C} 19=1$ & $0,26^{a}$ & $0,15^{\mathrm{b}}$ & $0,13^{\mathrm{bc}}$ & $0,09^{c}$ & 0,60 & 0,49 \\
\hline $\mathrm{C} 18=3$ & 0,44 & 0,47 & 0,43 & 0,49 & 0,19 & 0,25 \\
\hline$C 18=4$ & 0,46 & 0,53 & 0,44 & 0,53 & 0,20 & 0,40 \\
\hline $\mathrm{C} 20=1$ & 0,12 & 0,11 & 0,11 & 0,12 & 0,28 & 0,28 \\
\hline$\sum$ SAT pair & $27,47^{a}$ & $30,46^{\mathrm{b}}$ & $32,14^{c}$ & $35,58^{\mathrm{d}}$ & 0,78 & 0,08 \\
\hline$\sum$ INSAT $=1$ & $56,34^{\mathrm{a}}$ & $55,27^{\mathrm{a}}$ & $55,52^{\mathrm{a}}$ & $53,5^{\mathrm{b}}$ & 0,53 & 0,03 \\
\hline$\sum$ INSAT-C $18=1$ & $14,57^{\mathrm{a}}$ & $12,24^{b}$ & $10,39^{c}$ & $7,62^{d}$ & 0,75 & 0,21 \\
\hline $\bar{\Sigma}$ IMPRAM & $10,19^{a}$ & $8,37^{\mathrm{b}}$ & $7,11^{\mathrm{c}}$ & $5,55^{d}$ & 0,72 & 0,21 \\
\hline
\end{tabular}

$a, b, c, d$ : différences significatives à $p<0,05 ; \eta$ : coefficient de point ; c.v. : coefficient de variation ; $\Sigma$ IMPRAM : somme des acides gras impairs et/ou ramifiés; Ten 1 : gras très mou et huileux, Ten 4 : gras très ferme.

On remarque que sur cette base d'A.G., les différentes classes de notation sont assez bien discriminées. La régularité des distances entre classes sur le plan de projection de l'AFC, réalisée sur la base des profils d'A.G, confirme cette bonne séparation en particulier sur le premier axe principal représentant $74 \%$ de l'inertie totale (figure 1 ).
L'analyse discriminante fait aussi ressortir que $82,5 \%$ des échantillons restent bien classés à partir de leur composition en A.G.

Nous retrouvons pour l'essentiel les résultats obtenus à partir d'agneaux situés dans les classes extrêmes [4]. En opposition, bien que modestement impliqué, C18 $=1(\eta=0,58)$ présente des teneurs significativement dif- 
AFC

Fermeté \% de l'inertie

Ten $1\left(r^{2}\right)$

Ten2 $\left(r^{2}\right)$

Ten3 $\left(r^{2}\right)$

Ten4 $\left(r^{2}\right)$

Couleurs \% de l'inertie

Coul1 $\left(r^{2}\right)$

Coul2 $\left(r^{2}\right)$

Coul3 $\left(r^{2}\right)$

Coul4 $\left(r^{2}\right)$

Coul5 ( $\left.r^{2}\right)$

Coul6 ( $\left.\mathrm{r}^{2}\right)$

Coul7 $\left(r^{2}\right)$

\section{AD}

Axe 1

Axe 2

$\%$ de bien classés

$\begin{array}{cl}\text { Axe 1 } & \text { Axe 2 } \\ 74,1 \% & 6,4 \% \\ 0,99 & 0,002 \\ 0,62 & 0,32 \\ 0,78 & 0,06 \\ 0,98 & 0,01 \\ & \\ 72,1 \% & 6,3 \% \\ 0,96 & 0,00 \\ 0,97 & 0,00 \\ 0,30 & 0,18 \\ 0,97 & 0,00 \\ 0,94 & 0,00 \\ 0,22 & 0,45 \\ 0,14 & 0,04\end{array}$

\section{Fermeté Couleurs}

$63,2 \% \quad 35,5 \%$

$27,0 \% 26,4 \%$

$82,5 \% \quad 59,8 \%$
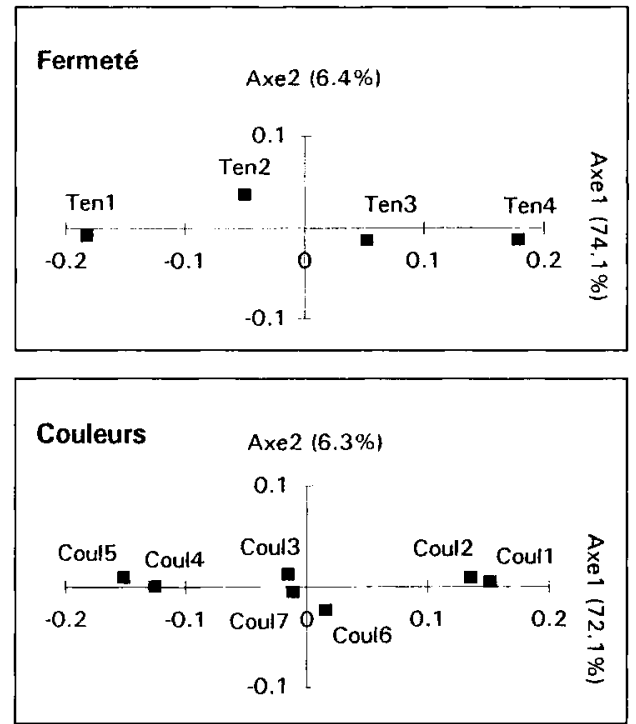

Figure 1. Analyses factorielles des correspondances (AFC) et discriminante (AD) selon les profils d'acides gras, la fermeté et la couleur des tissus gras sous-cutanés.

férentes entre classes $(p<0,05)$ sur cet échantillon caractérisé par des effectifs supérieurs et l'existence de classes intermédiaires. Cependant, la différence n'est que de $9 \%$ entre classes extrêmes. Sa forte représentation dans la composition $(>41 \%)$ et son point de fusion intermédiaire $\left(13,4{ }^{\circ} \mathrm{C}\right)$ en font un élément peu déterminant dans l'apparition du phénomène de non « caillage ». Ceci rejoint les résultats de Busboom et al. [8]. Contrairement à Molénat et Thériez [20], nous n'observons pas l'implication de l'A.G. : C18 = 2 .

Lorsqu' on se reporte au regroupement des A.G. saturés à nombre pair de carbones ( $\Sigma$ SAT pair), les différences sont très significativement marquées entre chaque degré de notation et la liaison avec la fermeté est assez bonne $(\eta=0,78)$. L'essentiel de la différence entre gras mous et gras fermes est apportée par les acides palmitique (C16) et stéarique $(\mathrm{C} 18)(\mathrm{C} 16+\mathrm{C} 18=24,4 \%$ vs $31,8 \%, p<0,05)$. Concernant la somme des A.G. mono-insaturés ( $\Sigma$ INSAT $=1$ ), les différences exprimées sont moins impor- tantes et la liaison est faible $(\eta=0,53)$. Seuls les gras les plus fermes possèdent une moyenne légèrement inférieure à celle des autres $(53,5 \%$ vs $55,7 \%, p<0,05)$. Ceci est à relier à la forte participation de l' acide oléique $(\mathrm{C} 18=1)$ dans cette somme ; celuici, à l'inverse des autres constituants, est présent en plus grande quantité dans les gras fermes. D'ailleurs, C11 = 1, C12=1, $\mathrm{C} 17=1$ possèdent un assez bon pouvoir discriminant $(\eta=0,75, \eta=0,80, \eta=0,74$ respectivement), bien qu'ils soient quantitativement faiblement représentés. En excluant $\mathrm{C} 18=1$ du regroupement, la liaison avec la fermeté est nettement améliorée, les gras mous et huileux étant deux fois plus riches en ces A.G. $(14,6 \%$ vs $7,6 \%, \eta=0,75)$.

Le regroupement A.G. impairs et/ou ramifiés ( $\Sigma$ IMPRAM) montre une tendance semblable $(10,2 \%$ vs $5,5 \%, p<0,001$; $\eta=0,72$ ).

Les gras très mous apparaissent significativement plus hydratés que les gras les plus fermes $(23,6 \%$ vs $17 \%, p<0,05$; 
tableau $I I)$. Cependant, le coefficient de variation est important, notamment pour Ten1 (c.v. : $26 \%$ ) et la liaison reste modeste $(\eta=0,51)$, rendant ce facteur peu discriminant. Ceci rejoint le constat de Molénat et Thériez [20] et confirme nos résultats antérieurs [3]. Par ailleurs, la fermeté ne paraît pas liée, dans cette étude, à l'importance du niveau de l'engraissement externe exprimé par les variables EGdo ( $p>0,35)$ et NGext $(p>0,37)$, la variabilité intra-classe étant très élevée notamment pour EGdo. En conséquence, les causes d'origine alimentaire semblent prépondérantes en raison de l'implication du métabolisme énergétique dans la genèse des acides gras. D'une part, le sevrage précoce favorise le stockage d'acides gras impairs et/ou ramifiés produits par les microorganismes du rumen par comparaison avec des agneaux allaités, d'autre part, les aliments fortement propio- géniques accentuent cette tendance en induisant la substitution de l'acide malonique par l'acide methylmalonique lors de la synthèse de novo des A.G., plus particulièrement dans le tissu gras sous-cutané $[9,10,13]$.

\subsection{Couleur du tissu gras externe}

Les liaisons entre composition en A.G. et notation de la couleur (tableau III) sont dans l'ensemble faibles $(\eta<0,63)$. Il en résulte que la discrimination à partir de la composition en A.G. du tissu gras souscutané est moins perceptible que dans le cas de la fermeté des tissus gras. Les teneurs des A.G : C10, C12, C14, IC17, C18 = 2, $\mathrm{C} 18=3, \mathrm{C} 18=4, \mathrm{C} 20=1$ ne sont pas significativement différentes selon les nuances de couleur.

Tableau II. Teneur en eau et état d'engraissement du tissu gras sous-cutané selon sa couleur et sa fermeté.

\begin{tabular}{lcccccccc}
\hline Fermeté & Ten1 & Ten2 & Ten3 & Ten4 & $\eta$ & & & \\
\hline Effectif & 20 & 20 & 20 & 20 & & & & \\
\% eau & $23,65^{\mathrm{a}}$ & $21,84^{\mathrm{ab}}$ & $20,44^{\mathrm{b}}$ & $16,98^{\mathrm{c}}$ & 0,51 & & & \\
c.v. & $26 \%$ & $18 \%$ & $17 \%$ & $15 \%$ & & & & \\
EGdo (mm) & 2,55 & 2,35 & 3,25 & 3,55 & 0,23 & & & \\
c.v. & $55 \%$ & $69 \%$ & $81 \%$ & $69 \%$ & & & & \\
NGext & 2,3 & 2,5 & 2,6 & 2,8 & 0,24 & & & \\
c.v. & $16 \%$ & $22 \%$ & $30 \%$ & $28 \%$ & & & & \\
\hline Couleur & Coul1 & Coul2 & Coul3 & Coul4 & Coul5 & Coul6 & Coul7 & $\eta$ \\
\hline Effectif & 16 & 16 & 16 & 16 & 16 & 16 & 16 & \\
$\%$ eau & $18,24^{\mathrm{b}}$ & $20,78^{\mathrm{ab}}$ & $20,23^{\mathrm{b}}$ & $24,55^{\mathrm{a}}$ & $20,77^{\mathrm{ab}}$ & $20,01^{\mathrm{b}}$ & $22,44^{\mathrm{ab}}$ & 0,41 \\
c.v. & $17 \%$ & $18 \%$ & $17 \%$ & $25 \%$ & $25 \%$ & $21 \%$ & $14 \%$ & \\
EGdo (mm) & 3,50 & 3,25 & 3,56 & 1,75 & 2,25 & 2,63 & 2,56 & 0,10 \\
c.v. & $62 \%$ & $71 \%$ & $78 \%$ & $44 \%$ & $50 \%$ & $77 \%$ & $64 \%$ & \\
NGext & $2,9^{\mathrm{a}}$ & $2,6^{\text {ab }}$ & $2,6^{\mathrm{ab}}$ & $2,2^{\mathrm{b}}$ & $2,5^{\mathrm{ab}}$ & $2,7^{\mathrm{ab}}$ & $2,1^{\mathrm{b}}$ & 0,39 \\
c.v. & $16 \%$ & $27 \%$ & $20 \%$ & $21 \%$ & $36 \%$ & $17 \%$ & $30 \%$ & \\
\hline
\end{tabular}

a. b.c : différences significatives à $p<0,05 ; \eta$ : coefficient de point ; c.v. : coefficient de variation.

Ten 1 : gras très mou et huileux, Ten $:$ gras mou, Ten $3:$ gras ferme, Ten $4:$ gras très ferme.

Coull : blanc, Coul 2 : blanc crème, Coul3 : rosé, Coul4 : bistre clair, Coul5 : brun-rouge, Coul6 : jaune clair, Coul7 : bistre clair avec reflêts verdâtres.

EGdo : épaisseur du gras dorsal en mm ; NGext : note de gras externe selon la grille Europa. 
Tableau III. Composition en acides gras selon la couleur du tissu gras sous-cutané.

\begin{tabular}{llllllllll}
\hline Couleur & Coul1 & Coul2 & Coul3 & Coul4 & Coul5 & Coul6 & Coul7 & $\eta$ & c.v. \\
\cline { 5 - 8 } & Blanc & $\begin{array}{c}\text { Blanc } \\
\text { crème }\end{array}$ & Rosé & $\begin{array}{c}\text { Bistre } \\
\text { clair }\end{array}$ & $\begin{array}{c}\text { Brun } \\
\text { rouge }\end{array}$ & $\begin{array}{c}\text { Jaune Bistre clair } \\
\text { clair avec reflets } \\
\text { verdâtres }\end{array}$ & \\
\hline
\end{tabular}

\begin{tabular}{|c|c|c|c|c|c|c|c|c|c|}
\hline Effectif & 16 & 16 & 16 & 16 & 16 & 16 & 16 & & \\
\hline \multicolumn{10}{|l|}{ Acides gras } \\
\hline $\mathrm{C} 10$ & 0,24 & 0,21 & 0,23 & 0,21 & 0,22 & 0,21 & 0,21 & 0,22 & 0,27 \\
\hline $\mathrm{C} 10=1$ & $0,16^{c}$ & $0,18^{c}$ & $0,26^{\mathrm{ab}}$ & $0,31 \mathrm{a}$ & $0,32^{\mathrm{a}}$ & $0,23^{b}$ & $0,28^{\mathrm{ab}}$ & 0,49 & 0,34 \\
\hline $\mathrm{C} 11=1$ & $0,03^{\mathrm{c}}$ & $0,06^{c}$ & $0,17^{\mathrm{ab}}$ & $0,26^{\mathrm{a}}$ & $0,3^{\mathrm{a}}$ & $0,15^{\mathrm{b}}$ & $0,16^{\mathrm{b}}$ & 0,58 & 0,79 \\
\hline $\mathrm{C} 12$ & 0,20 & 0,16 & 0,18 & 0,16 & 0,16 & 0,16 & 0,16 & 0,22 & 0,43 \\
\hline $\mathrm{C} 12=1$ & $0,07^{\mathrm{c}}$ & $0,08^{\mathrm{c}}$ & $0,13^{\mathrm{b}}$ & $0,17^{\mathrm{a}}$ & $0,18^{a}$ & $0,12^{b}$ & $0,14^{\mathrm{ab}}$ & 0,62 & 0,40 \\
\hline $\mathrm{IC} 13$ & $0,07^{\mathrm{c}}$ & $0,09^{\mathrm{c}}$ & $0,21^{\mathrm{b}}$ & $0,29^{a}$ & $0,30^{\mathrm{a}}$ & $0,19^{\mathrm{b}}$ & $0,23^{\mathrm{ab}}$ & 0,62 & 0,55 \\
\hline $\mathrm{C} 13$ & $0,05^{\mathrm{c}}$ & $0,05^{c}$ & $0,10^{\mathrm{ab}}$ & $0,13^{a}$ & $0,15^{\mathrm{a}}$ & $0,09^{b}$ & $0,10^{\mathrm{ab}}$ & 0,59 & 0,51 \\
\hline IC14 & $0,04^{d}$ & $0,09^{c d}$ & $0,36^{\mathrm{ab}}$ & $0,51^{\mathrm{ab}}$ & $0,57^{\mathrm{a}}$ & $0,30^{\mathrm{bc}}$ & $0,32^{\mathrm{bc}}$ & 0,42 & 1,04 \\
\hline $\mathrm{C} 14$ & 3,31 & 3,01 & 2,99 & 2,78 & 2,92 & 3,05 & 2,88 & 0,26 & 0,20 \\
\hline $\mathrm{C} 14=1$ & $1,34^{\mathrm{d}}$ & $1,33^{\mathrm{d}}$ & $1,85^{\mathrm{bc}}$ & $2,30^{\mathrm{a}}$ & $2,29^{\mathrm{a}}$ & $1,71^{\mathrm{b}}$ & $1,92^{\mathrm{ab}}$ & 0,57 & 0,30 \\
\hline IC15 & $0,18^{c}$ & $0,19^{c}$ & $0,38^{b}$ & $0,46^{\mathrm{ab}}$ & $0,53^{\mathrm{a}}$ & $0,35^{\mathrm{b}}$ & $0,36^{\mathrm{b}}$ & 0,59 & 0,49 \\
\hline AIC15 & $0,21^{\mathrm{c}}$ & $0,22^{\mathrm{c}}$ & $0,33^{b}$ & $0,41^{\mathrm{a}}$ & $0,44^{\mathrm{a}}$ & $0,32^{b}$ & $0,34^{\mathrm{b}}$ & 0,63 & 0,31 \\
\hline $\mathrm{C} 15$ & $1,00^{\mathrm{c}}$ & $1,00^{c}$ & $1,38^{\mathrm{b}}$ & $1,70^{\mathrm{a}}$ & $1,88^{\mathrm{a}}$ & $1,35^{\mathrm{b}}$ & $1,39^{\mathrm{b}}$ & 0,61 & 0,30 \\
\hline $\mathrm{C} 15=1$ & $0,23^{c}$ & $0,26^{c}$ & $0,71^{\mathrm{b}}$ & $1,05^{\mathrm{a}}$ & $1,24^{\mathrm{a}}$ & $0,59^{b}$ & $0,68^{\mathrm{b}}$ & 0,52 & 0,67 \\
\hline IC16 & $0,23^{\mathrm{c}}$ & $0,22^{\mathrm{c}}$ & $0,46^{\mathrm{bc}}$ & $0,57^{\mathbf{b}}$ & $0,71^{\mathrm{a}}$ & $0,40^{c}$ & $0,41^{\mathrm{c}}$ & 0,62 & 0,50 \\
\hline $\mathrm{C} 16$ & $21,90^{\mathrm{a}}$ & $21,30^{\mathrm{a}}$ & $19,63^{\mathrm{ab}}$ & $18,86^{\mathrm{b}}$ & $19,25^{\mathrm{b}}$ & $20,87^{\mathrm{a}}$ & $19,91^{\mathrm{ab}}$ & 0,52 & 0,09 \\
\hline $\mathrm{C} 16=1$ & $4,29^{c}$ & $4,45^{c}$ & $5,08^{\mathrm{b}}$ & $5,86^{\mathrm{a}}$ & $5,72^{\mathrm{a}}$ & $5,06^{\mathrm{b}}$ & $5,23^{\mathrm{ab}}$ & 0,60 & 0,15 \\
\hline IC 17 & 0,25 & 0,25 & 0,30 & 0,27 & 0,31 & 0,29 & 0,28 & 0,31 & 0,22 \\
\hline AIC 17 & $0,86^{c}$ & $0,89^{c}$ & $1,20^{\text {ab }}$ & $1,30^{\mathrm{a}}$ & $1,31^{\mathrm{a}}$ & $1,08^{\mathrm{b}}$ & $1,14^{b}$ & 0,62 & 0,20 \\
\hline $\mathrm{C} 17$ & $2,68^{b}$ & $2,50^{\mathrm{bc}}$ & $3,03^{\mathrm{ab}}$ & $3,16^{\mathrm{a}}$ & $3,36^{\mathfrak{a}}$ & $2,64^{b}$ & $2,76^{\mathrm{b}}$ & 0,41 & 0,19 \\
\hline $\mathrm{C} 17=1$ & $1,84^{c}$ & $2,04^{c}$ & $2,72^{b}$ & $3,57^{\mathrm{a}}$ & $3,55^{\mathrm{a}}$ & $2,57^{\mathrm{a}}$ & $2,74^{b}$ & 0,60 & 0,32 \\
\hline IC18 & $0,26^{d}$ & $0,26^{\mathrm{d}}$ & $0,48^{\mathrm{b}}$ & $0,56^{a b}$ & $0,62^{a}$ & $0,39^{\mathrm{bc}}$ & $0,42^{\mathrm{bc}}$ & 0,61 & 0,40 \\
\hline $\mathrm{C} 18$ & $9,46^{\mathrm{a}}$ & $9,16^{\mathrm{a}}$ & $7,85^{\mathrm{b}}$ & $6,64^{c}$ & $6,71^{\mathrm{bc}}$ & $7,37^{\mathrm{bc}}$ & $7,45^{\mathrm{bc}}$ & 0,53 & 0,22 \\
\hline $\mathrm{C} 18=1$ & $45,67^{\mathrm{a}}$ & $45,52^{\mathrm{a}}$ & $44,05^{\mathrm{ab}}$ & $43,10^{\mathrm{bc}}$ & $41,41^{c}$ & $44,58^{\mathrm{ab}}$ & $44,54^{a b}$ & 0,53 & 0,06 \\
\hline $\mathrm{C} 18=2$ & 4,17 & 4,19 & 4,47 & 4,03 & 4,02 & 4,52 & 4,51 & 0,27 & 0,16 \\
\hline C19 & $0,17^{\mathrm{ab}}$ & $0,16^{\mathrm{b}}$ & $0,20^{\mathrm{a}}$ & $0,20^{\mathrm{a}-}$ & $0,19^{\mathrm{ab}}$ & $0,16^{b}$ & $0,17^{a b}$ & 0,33 & 0,21 \\
\hline $\mathrm{C} 19=1$ & $0,09^{d}$ & $0,11^{\mathrm{cd}}$ & $0,15^{\mathrm{bc}}$ & $0,22^{a}$ & $0,19^{a b}$ & $0,14^{\mathrm{cb}}$ & $0,16^{\mathrm{cb}}$ & 0,48 & 0,51 \\
\hline $\mathrm{C} 18=3$ & 0,48 & 0,43 & 0,46 & 0,38 & 0,43 & 0,47 & 0,42 & 0,28 & 0,26 \\
\hline $18=4$ & 0,40 & 0,50 & 0,52 & 0,47 & 0,44 & 0,51 & 0,57 & 0,22 & 0,48 \\
\hline $20=1$ & 0,11 & 0,11 & 0,12 & 0,11 & 0,11 & 0,11 & 0,11 & 0,17 & 0,21 \\
\hline SAT pair & $35,12^{\mathrm{a}}$ & $33,83^{\mathrm{ab}}$ & $30,8^{c}$ & $28,64^{c}$ & $29,25^{\mathrm{c}}$ & $31,66^{\mathrm{bc}}$ & $30,61^{\mathrm{c}}$ & 0,58 & 0,10 \\
\hline$\sum$ INSAT $=1$ & $53,83^{\mathrm{b}}$ & $55,12^{\mathrm{ab}}$ & $55,25^{\mathrm{ab}}$ & $56,93^{a}$ & $55,3^{\mathrm{ab}}$ & $55,27^{\mathrm{ab}}$ & $55,98^{a b}$ & 0,38 & 0,04 \\
\hline$\sum$ INSAT $-\mathrm{C} 18=1$ & $18,17^{c}$ & $8,61^{\mathrm{c}}$ & $11,2^{b}$ & $13,83^{\mathrm{a}}$ & $13,88^{\mathrm{a}}$ & $10,69^{\mathrm{b}}$ & $11,44^{\mathrm{b}}$ & 0,62 & 0,24 \\
\hline$\sum$ IMPRAM & $6,79^{\mathrm{d}}$ & $6,71^{d}$ & $9,47^{b c}$ & $10,85^{\mathrm{ab}}$ & $11,82^{a}$ & $8,60^{\mathrm{cd}}$ & $8,97^{b c}$ & 0,61 & 0,26 \\
\hline
\end{tabular}

a, h.c. d : différences significatives à $p<0,05 ; \eta$ : coefficient de point ; c.v. : coefficient de variation.

$\Sigma$ IMPRAM : somme des acides gras impairs et/ou ramifiés. 
Au vu des profils en A.G., les couleurs claires (Coul1 et Coul2) peuvent être associées. Celles-ci sont notamment plus riches en $\mathrm{C} 18$, et plus pauvres en A.G. à faible point de fusion (impair et/ou ramifiés, et /ou mono-insaturés) que les autres classes $(p<0,05)$. C'est l'inverse avec les nuances Coul4 et Coul5. De plus, la nuance brunrouge (Coul5) est associée à la plus faible teneur en $\mathrm{C} 18=1$. Les autres colorations se situent en position intermédiaire.

Ces observations rejoignent nos propres constats ainsi que ceux de Busboom et al. [8]. Celles-ci correspondent également aux données de Prache et al. [22] en ce qui concerne les acides gras impairs et ramifiés, mais divergent sensiblement pour les A.G. mono-insaturés et saturés, notamment pour la position relative de la teinte jaune. Zhou et al. [28] indiquent une liaison étroite entre $\mathrm{C} 18=1$ et la pigmentation jaune liée à la présence de caroténoïdes chez les bovins. Ceci ne semble pas être le cas chez les ovins. D'ailleurs, dans cette espèce, et contrairement aux bovins, la présence de caroténoïdes semble absente sous la forme de carotène et très faible sous la forme de lutéine [27]. Pourtant, Prache et al. [22] relatent des teneurs de 30 à 300 fois supérieures.

Les différences de composition en A.G. impairs et/ou ramifiés selon les types de couleurs sont les plus marquées, mais la participation factorielle à la variabilité reste tout de même assez faible $(\eta=0,61)$. Les tissus gras blancs à blanc crème se sont révélés les moins riches à l'opposé des gras brunrouges $(6,7 \%$ v.s. $11,8 \%, p<0,05)$. Les autres catégories se situent en position intermédiaire.

Concernant les A.G. mono-insaturés, peu de différence apparaît si ce n'est la plus faible teneur pour les gras blancs et la plus forte concentration pour les gras bistre clair $(53,8 \%$ v.s. $56,9 \%, p<0,05)$. Cependant, si ce regroupement n'associe pas $\mathrm{C} 18=1$, on retrouve un profil parallèle à celui des A.G. impairs et /ou ramifiés $(\eta=0,62)$. Ce résultat conforte l'hypothèse d'une plus grande fragilité à la peroxydation de cette catégorie comme le suggérait l'effet positif d'une supplémentation en vitamine $\mathrm{E}$ pour cette même nuance [6]. Il est probable que cette coloration survienne sous l'effet de la production de radicaux libres responsables d'une fragilisation des hématies chez des animaux génétiquement sensibles (variation individuelle et influence paternelle) comme l'indiquent Thériez et al. [24]. Les dérivés hémoglobiniques pourraient alors agir comme des catalyseurs de l'oxydation des A.G. insaturés [7].

Dans le cas des nuances claires, la plus forte proportion d'A.G. saturés pairs participe à l'association souvent constatée entre gras fermes et gras blancs. Cependant, compte tenu de la modeste contribution du facteur couleur pour expliquer la variation des teneurs en A.G. $(\eta=0.58)$, il en découle que l'hypothèse explicative soit plutôt d'ordre multifactoriel.

Sur la base de la composition en A.G., l'analyse factorielle des correspondances (figure I) fait ressortir la proximité entre Coull et Coul 2 d'une part et Coul4 et Coul5 d'autre part. Les autres classes ne sont pas suffisamment bien représentées pour conclure. L'analyse discriminante conforte ce point de vue en ne permettant de classer correctement que $59,8 \%$ des échantillons.

S'agissant de la teneur en eau (tableau $I I)$, nous retrouvons l'opposition déjà constatée entre Coul1 et Coul4 : plus faible teneur pour les gras blancs et plus forte pour les gras bistres clairs ( $18,24 \%$ v.s. $24,5 \%$, $p<0,05$ ), les autres couleurs se situant en position intermédiaire. Cette particularité pourrait résulter en partie d'une lipolyse couplée à une réaction d'auto-oxydation post mortem génératrice de molécules $\mathrm{H}_{2} \mathrm{O}$ [18] renforçant la teneur en eau du tissu gras sous-cutané. Cette lipolyse pourrait être préalablement initiée par une susceptibilité au stress (transport, conditions d'abattage) sous l'effet de la production de catécholamines. L'hypothèse d'un état de finition moins avancé peut-être également propo- 
sée. En effet, pour ce type d'agneaux, deux populations d'adipocytes coexistent, l'une de petite taille cellulaire, l'autre au stade de l'hypertrophie cellulaire associée à l'accrétion [23]. Dans ces conditions, le contenu cellulaire des adipocytes juvéniles est encore riche en eau et l'activité enzymatique très développée dans un contexte d'abaissement de la température et de ralentissement de la chute du $\mathrm{pH}$. La tendance pour cette catégorie à présenter des carcasses moins grasses (EGdo : $1,75 \mathrm{~mm}$ vs $3,5 \mathrm{~mm}(p<0,09)$, Ngext : 2,2 vs $2,9(p<0,05))$ conforte cette possibilité.

Hormis cette couleur particulière, la liaison de la teneur en eau avec le développement des colorations est encore plus faible que dans le cas de la fermeté $(\eta=0,41)$.

En conséquence, la composition du tissu gras en A.G. semblerait n'être qu'un support sur lequel d'autres phénomènes pourraient se greffer. Le cas des oxydations post mortem pour l'apparition de la couleur bistre clair en est une illustration. De même, l'accroissement des quantités de lutéines ainsi que leur plus grande facilité d'absorption en présence d'A.G. alimentaires désaturés ayant échappé à l'hydrogénation ruminale [1] ou de dérivés hémoglobiniques [22] sont à envisager pour les colorations jaunes. Dans le cas des colorations brun-rouge, en plus de la forte proportion en A.G. ramifiés et/ou impairs à bas point de fusion, nous remarquons, à l'inverse, presque en substitution, la plus faible proportion d'acide oléique. Bien qu'insaturé, cet A.G. long possède un point de fusion (notamment pour la forme trans non séparée dans cette analyse) qui s'apparente à celui des A.G. longs et favorise le « caillage » des gras de carcasses refroidies [27]. Aussi, cette particularité peut-elle rendre compte de l'association souvent constatée entre ce type de coloration et le caractère mou et huileux du tissu gras. Pour cette catégorie, une participation génétique, en particulier chez les jeunes agneaux mâles à forte vitesse de croissance et faible niveau d'engraissement [4], pourrait être incriminée. En effet, dans le cas d'une sélection sur ce critère, l'apport énergétique du régime alimentaire peut-être insuffisant et induire indirectement un déficit protéique en raison de l'utilisation glucogénique des acides aminés. Dès lors, l'implication de G.H. dans la régulation de l'activité métabolique [26] pourrait induire une réduction de l'activité lipogénique, notamment des élongases, rendant compte des plus faibles proportions de $\mathrm{C} 16$ et $\mathrm{C} 18$, et dans une moindre mesure de la $\Delta 9$ désaturase, comme l'ont montré chez le rat Narce et al. [21], conduisant du C18 au C18 = 1 dans le tissu gras, site le plus actif chez les ruminants [25].

\subsection{Relation entre fermeté et couleur du tissu sous-cutané}

Le tableau $I V$ de contingence entre degré de fermeté et type de coloration observés sur 570 agneaux et la figure 2, illustratrice de l'AFC correspondante, font ressortir les éléments suivants :

- la proportion de carcasses défectueuses tant sur le plan de la fermeté (Ten1 + Ten2 : $34 \%$ ) que sur celui de la couleur (Coul3, $4,5,6,7: 49,6 \%$ ) est importante, ce qui rejoint les observations déjà mentionnées à ce sujet $[12,16]$;

- les couleurs claires (blanc, blanc crème) sont plus souvent associées aux tenues fermes (Ten4 et Ten3). Cependant, le lien n'est pas très étroit et la dispersion des notations est assez forte. À classe de fermeté égale uñ grand nombre de nuances de coloration peut se développer. La représentation à partir des deux premiers axes principaux de l'AFC rend compte de $90 \%$ de l'inertie totale. L'axe 1 oppose les tissus très fermes (Ten4) contribuant pour $59 \%$ à la formation de cet axe et les tenues molles (Ten 1 et Ten2 39,6\%). En parallèle, la coloration blanche contribue pour $64 \%$ à cet axe. L'axe 2 est associé aux gras de couleur brunrouge et aux gras très mous et huileux. Sur ce plan, l'association entre tenue ferme et 
Tableau IV. Tableau de contingence des effectifs observés exprimés en \%, selon les classes de fermeté et de couleur du tissu gras sous-cutané $(n=570)$

\begin{tabular}{lllllllll}
\hline Couleur & Coul1 & Coul2 & Coul3 & Coul4 & Coul5 & Coul6 & Coul7 & Total \\
& Blanc & $\begin{array}{c}\text { Blanc } \\
\text { crème }\end{array}$ & Rosé & $\begin{array}{c}\text { Bistre } \\
\text { clair }\end{array}$ & $\begin{array}{c}\text { Brun } \\
\text { rouge }\end{array}$ & $\begin{array}{c}\text { Jaune } \\
\text { clair }\end{array}$ & $\begin{array}{c}\text { Bistre clair } \\
\text { avec reflets } \\
\text { verdâtres }\end{array}$ & \\
& & & & & & & &
\end{tabular}

\section{Fermeté}

\begin{tabular}{|c|c|c|c|c|c|c|c|c|}
\hline Ten 1 & 0,0 & 0,4 & 1,4 & 1,2 & 1,4 & 0,9 & 0,0 & 5,3 \\
\hline Ten2 & 0,5 & 6,8 & 6,8 & 9,0 & 0,7 & 2,8 & 2,3 & 29,0 \\
\hline Ten 3 & 8,4 & 23,0 & 7,9 & 7,9 & 1,1 & 3,7 & 1,1 & 53,0 \\
\hline Ten 4 & 7,7 & 3,5 & 0,5 & 0,4 & 0,2 & 0,3 & 0,2 & 12,8 \\
\hline Total & 16,7 & 33,7 & 16,7 & 18,4 & 3,2 & 7,7 & 3,5 & \\
\hline
\end{tabular}

Ten 1 : gras très mou et huileux, Ten $2:$ gras mou, Ten $3:$ gras ferme, Ten $4:$ gras très ferme.

\section{Axe 1 Axe 2 Axe 3}

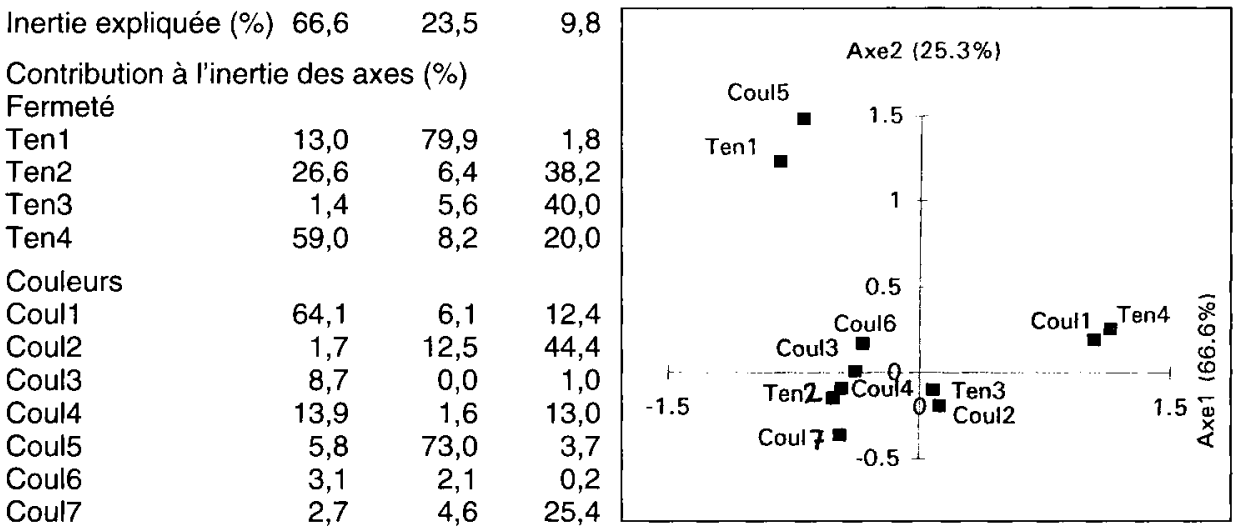

Figure 2. Analyse factorielle des correspondances selon la fermeté et la couleur des tissus gras souscutanés.

couleur blanche ainsi que tenue molle et huileuse et couleur brun-rouge paraît confirmée. La liaison entre couleur blanc crème et Ten 3 est mieux représentée par l'axe 3 . Les autres caractéristiques ne sont pas suffisamment liées ou sont mal représentées dans cette analyse.

\section{CONCLUSION}

Les résultats issus de l'analyse de la composition en A.G. des triglycérides du tissu gras sous-cutané valident pour l'essentiel les conclusions déjà présentées sur ce sujet. La qualité des triglycérides constitutifs reste le principal facteur explicatif des variations de la fermeté du gras de couverture. Cependant, l'appréciation de ce critère qualitatif est à l'origine d'un biais non négligeable lié, en particulier, à la température ambiante du lieu où se pratique le jugement (réchauffement des carcasses sorties des chambres froides). L'amélioration de la fermeté semble, en conséquence, devoir reposer sur la maî- 
trise qualitative de l'adipogénèse et la recherche de la précocité ainsi que sur la prise en compte des conditions de la chaîne du froid lors du traitement des carcasses.

Il n'en va pas de même pour l'expression de la coloration du tissu gras externe. Les faibles liaisons enregistrées confortent l'hypothèse d'une causalité multiple où les suspicions peuvent porter sur l'interaction de facteurs d'ordre génétiques - liés à la croissance, à l'efficacité à assimiler et fixer la lutéine [2], à la sensibilité à l'hémolyse [24] - comme le révèle l'effet race [17], d'ordre oxydatif durant la phase de ressuage en cas de carence d'antioxydants ou bien d'ordre pathologique à propos des rares cas d'apparition d'ictères prononcés. Une incidence moins fortuite, rattachée à la prévalence du parasitisme interne ordinaire (coccidioses pour les agneaux de bergerie, strongyloses pour les agneaux d'herbage) serait également à retenir. En tout état de cause, la composition en acides gras du tissu adipeux ne peut expliquer la diversité des couleurs des carcasses d'agneaux.

\section{RÉFÉRENCES}

11] Ashes J.R., Burley R.W, Sidhu G.S, Sleigh R.W., Effect of particule size and lipid composition of bovine blood high density lipoprotein on its function as a carrier of $\beta$-carotene, Biochim. Acla 797 (1984) 171-177.

12] Baker R.L., Steine T., Vabeno A.W., Breines D., The inheritance and incidence of yellow fat in norwegian sheep, Acta Agric. Scand. 35 (1985) 389-397.

131 Bouillier-Oudot M., Bozzolo G., Grasset D., Manse H., Optimisation du jugement de la fermeté du gras de couverture des carcasses d'agneaux de bergerie, Ann. Zootech. 41 (1992) 187-203.

41 Bozzolo G., Bouillier-Oudot M., De Boisseson E., Ghassan M., Grasset D., Influence des performances zootechniques sur les caractéristiques des lissus adipeux d'agneaux de bergerie, sevrés précocement et alimentés avec un régime à forte concentration énergétique, Ann. Zootech. 39 (1990) 77-94.

[5] Bozzolo G., Bouillier-Oudot M., Phrem G., Grasset D., Manse H.. Effet de l'incorporation de saindoux dans le concentré d'engraissement sur la croissance et la qualité des carcasses chez l'agneau de bergerie, Cah. Agric. 2 (1993) $394-405$.

|6] Bozzolo G., Bouillier-Oudot M., Phrem G., Effet d'une surcharge en vitamine $\mathrm{E}$, administrée oralement avant abattage, sur la coloration du gras de couverture des carcasses d'agneaux de bergerie, Ann. Zootech. 42 (1993) 67-73.

[7] Buckley D.J., Gray J.I., Asghar A., Price J.F., Crackel R.L., Booren A.M., Pearson A.M., Miller E.R., Effects of dietary antioxidants and oxidised oil on membranal lipid stability and pork product quality, J. Food Sci. 54 (1989) 1193-1197.

[8] Busboom J.R., Miller G.J., Field R.A., Crouse J.D., Riley M.L., Nelms G.E., Ferell C.L., Characteristics of fat from heavy ram and wether lambs, J. Anim. Sci. 52 (1981) 83-92.

[9] Christie W.W., The effects of diet and other factors on the lipid composition of ruminant tissues and milk, in: Christie W.W. (Ed.), Lipid Metabolism in Ruminant Animals, Pergamon Press Publ, Oxford, UK, 1989, pp. 193-225.

[10] Church D.C., Metabolism of lipids, in: Digestive Physiology and Nutrition of Ruminants: Nutrition, Oxford Press, Portland, OR, USA, vol. 2 , 1979, pp. 258-263.

[11] Dagnélie P., Théorie et méthodes statistiques vol 1, Gembloux : Les Presses agronomiques de Gembloux, 1979, 378 p.

[12] De Boisseson E., Mahmoudi G., Qualité du gras de l'agneau de roquefort, mémoire de fin d'études Ensat, non publié, 1989, 48 p.

[13] Folch J., Lees M., Sloane Stanley G.H., A simple method for the isolation and purification of total lipids from animal tissues, J. Biol. Chem. 226 (1957) 497-509.

114] Garton G.A., Howell F.D.D., Duncan W.R.H., Influence of dietary volatile fatty acids on the fatty acid composition of lamb triglycerides with special reference to the effect of propionate on the presence of branched chain components, $\mathrm{Br}$. J. Nutr. 28 ( 1972) 409-416.

[15] Jenkins T.C., Regulation of lipid metabolism in the rumen, J. Nutr. 124 (1994) 1372S-1376S.

[16] Legrand I., Qualité du gras, Pâtre 441 (1994) $21-27$.

[17] Legrand I., Influence de la race et du rationnement sur la qualité du gras des agneaux, Renc. Rech. Ruminants 1 (1994) 209-212.

18] Lchninger A.L., Les enzymes d'oxydoréduction et les transports d'électrons, in : Biochimic, Flammarion Médecine Sciences, 1979. pp. 471-502.

119] Miller G.J., Rice R.W., Lipid metabolism in lambs as affected by fattening rations of roughage and concentrate, J. Anim. Sci. 26 (1967) $1153-1159$.

[20] Molénat G., Théricz M., Influence du mode d'ćlevage sur la qualité de la carcasse de l'agneau de bergerie, Ann. Zootech. 22 (1973) $279-293$. 
[21] Narce M., Poisson J.P., Belleville J., Chanussot B. Depletion of $\Delta 9$ desaturase (EC 1.14.99.5) enzyme activity in growing rat during dietary protein restriction, Br. J. Nutr. 68 (1992) 627-637.

[22] Prache S., Aurousseau B., Thériez M., Renerre M., Les défauts de couleur du tissu adipeux souscutané des carcasses d'ovins, Inra Prod. Anim. 3 (1990) 275-285.

[23] Smith S.B., Jenkins T., Prior R.L., Carcass composition and adipose tissue metabolism in growing sheep, J. Anim. Sci. 65 (1987) 1525-1530.

[24] Thériez M., Aurousseau B., Prache S., Mendizabal J., Les défauts de couleur des gras d'agneaux, Renc. Rech. Ruminants 4 (1997) 295-301.
[25] Wahle K.W.J., Desaturation of long-chain fatty acids by tissue preparations of the sheep, rat and chickens, Comp. Biochem. Physiol. 43B (1974) 87-105.

[26] Waghorn G.C., Flux D.S., Ulyatt M.J., Effects of dietary protein and energy intakes on growth hormone, insulin, glucose tolerance and fatty acid synthesis in young wether sheep, Anim. Prod. 44 (1987) 143-152.

[27] Yang A., Larsen T.W., Tume R.K., Carotenoid and retinol concentrations in serum, adipose tissue and liver and carotenoid transport in sheep, goats and cattle, Aust. J. Agric. Res. 43 (1992) 1809-1817.

[28] Zhou G.H., Yang A., Tule R.K., A relationship between bovine fat colour and fatty acid composition, Meat Sci. 35 (1993) 205-212. 